\title{
Quantum Fisher Information of Two-Level Atomic System under the Influence of Thermal Field, Intrinsic Decoherence, Stark Effect and Kerr-Like Medium
}

\author{
S. Jamal Anwar, M. Ramzan, M. Usman, M. Khalid Khan \\ Department of Physics, Quaid-i-Azam University, Islamabad, Pakistan \\ Email: sjamalanwar@gmail.com
}

How to cite this paper: Anwar, S.J., Ramzan, M., Usman, M. and Khan, M.K. (2021) Quantum Fisher Information of Two-Level Atomic System under the Influence of Thermal Field, Intrinsic Decoherence, Stark Effect and Kerr-Like Medium. Journal of Quantum Information Science, 11, 24-41.

https://doi.org/10.4236/jqis.2021.111003

Received: December 14, 2020

Accepted: March 13, 2021

Published: March 16, 2021

Copyright $\odot 2021$ by author(s) and Scientific Research Publishing Inc. This work is licensed under the Creative Commons Attribution International License (CC BY 4.0).

http://creativecommons.org/licenses/by/4.0/

(c) (i) Open Access

\begin{abstract}
In this paper, we have proposed the numerical calculations to study the quantum entanglement $(\mathrm{QE})$ of moving two-level atom interacting with a coherent and the thermal field influenced by intrinsic decoherence (ID), Kerr medium (non-linear) and the Stark effect. The wave function of the complete system interacting with a coherent and the thermal field is calculated numerically affected by ID, Kerr (non-linear) and Stark effects. It has been seen that the Stark, Kerr, ID and the thermal environment have a significant effect during the time evolution of the quantum system. Quantum Fisher information (QFI) and QE decrease as the value of the ID parameter is increased in the thermal field without the atomic movement. It is seen that QFI and von Neumann entropy (VNE) show an opposite and periodic response in the presence of atomic motion. The non-linear Kerr medium has a more prominent and significant effect on the QE as the value of the Kerr parameter is decreased. At smaller values of the non-linear Kerr parameter, the VNE increases, however, QFI decreases, so QFI and VNE have a monotonic connection with one another. As the value of the Kerr parameter is increased, the effect of non-linear Kerr doesn't stay critical on both QFI and QE. However, a periodic response of $\mathrm{QE}$ is seen because of the atomic movement which becomes modest under natural impacts. Moreover, it has been seen that QFI and QE rot soon at the smaller values of the Stark parameter. However, as the value of the Stark parameter is increased, the QFI and QE show periodic response even when the atomic movement is absent.
\end{abstract}

\section{Keywords}

Quantum Fisher Information (QFI), Intrinsic Decoherence (ID), Thermal Environment, Two-Level Atomic System, Kerr and Stark Effect 


\section{Introduction}

Quantum metrology (QM) is the most important and developing field of modern research in quantum information theory (QIT) [1] [2] [3] [4]. Quantum mechanics gives a new breakthrough to the field of parameter estimation [5] [6]. The main objective of quantum mechanics is to explore the restrictions of the parameter-estimation limited by the laws of quantum mechanics. Quantum mechanics has a large number of applications such as improving the time and frequency standards [7] [8], detection of gravitational waves [9], and synchronization of clock [10], etc. Quantum Fisher information (QFI) has a key part in quantum mechanics. Quantum Fisher Information (QFI) is a very fascinating and interesting idea thought for the examination of the situations that need phase sensitivity. Quantum Fisher information (QFI) has great importance for quantum estimation theory and QIT [11] [12] [13] [14]. In the quantum estimation theory, the main objective is to compute the value of an unknown parameter related to the quantum system, and the primary aim is to increase the precision and accuracy [15] [16] [17] [18]. The inverse of the QFI provides the lower bound of error of the estimation [19] [20]. Hence, how to improve the QFI turns into the basic problem to be resolved. Also, statistical distinguishability is measured with the help of QFI on the space of the density operators in the quantum information geometry [21] [22]. Recently, the QFI flow was suggested as a quantitative measure of the information flow and provided an innovative point of view on studying the non-Markovian behaviour in open quantum systems [23].

Different quantum entanglement (QE) measures for pure states have been suggested, like von Neumann entropy (VNE) [24] [25], concurrence [26] [27] and linear entropy [28], etc. The relationship between the QE and Fisher information (FI) has not been investigated extensively. In this context, the QE evaluation is calculated using atomic Fisher information (AFI) [29]. It has been observed that QE of a two-level atom can be determined by using AFI and its marginal distribution. In addition, the QFI gives an acceptable condition to observe multi-particle QE in an N qubit state [12]. Another use of the FI is to investigate polarization squeezing and multipartite $\mathrm{QE}$ of a quantum light field. Moreover, the FI determines the correlation between the quantized field and Kerr medium [30].

The interaction between the electromagnetic field and matter is a major quantum optical problem and it has a large number of applications in laser physics and quantum optics [31] [32]. The most simplified model in the atom optics is well-know Jaynes-Cummings model (JCM) [33] which describes the interaction between a single two-level atom and the quantized field in a rotating wave approximation (RWA). This model has been explored to a great extent before [34]. A number of generalized models have been studied [35]. Multiphoton and multimode processes can be discussed through these generalizations from a theoretical point of view [36]. However, in recent years, researches have strongly focused on the nonlinear interaction between a two-level atom and field in the 
deformed JCM. The JCM has been investigated under the effect of Stark-shift and Kerr like medium [37] [38]. Abdel-Aty et al. [39] discussed the entropy evolution of the bimodal field interacting with a two-level atom in the presence of the Kerr medium. The interaction of a three-level atom with a single-mode cavity field having intensity-dependent coupling in a Kerr medium has been studied [40]. Abdalla et al. studied the interaction of a two-level atom with a single-mode multi-photon field in the medium including the Stark shift and Kerr medium effects with the coupling term which is supposed to be a function of time, but still linear with the intensity of light [41]. Besides, it is partially nonlinear only due to the effect of the Kerr medium. Anyhow, in the last few years, the effect of the atomic motion and field-mode structure on QE has become attractive for researchers [42].

Real quantum systems always have the effect of the surrounding environment. Decoherence is generated because of the interaction between the environment and quantum systems and hence it can also induce QE. Therefore, the QE always arises in the interaction of an arbitrary big system in any mixed state with a single qubit in the pure state. The effect of intrinsic decoherence in quantum systems is discussed in a large number of papers [43] [44]. One explanation of why quantum coherences are decreased and difficult to observe that the closed quantum systems don't evolve unitarily according to the Schrodinger equation, but are influenced by more generalized equations that include ID. In particular, a classic model of ID based on a simple change of a unitary Schrodinger evolution has been suggested. This model is established on the hypothesis that on adequately short time scales the closed system grows by an irregular arrangement of unitary phase changes developed by the system Hamiltonian.

In the last few years, researchers focused on the QE and QFI of a two-level atomic system as it can be considered as one qubit system. A.-S. F. Obada studied the QE evolution with atomic Fisher information (AFI) [29]. S. Abdel-Khalek and his fellows studied the QFI of a single-qubit system [45]. K.Berrada and co-workers investigated the QFI of the qubit system placed inside the dissipative cavity [46]. Buzek studied the effect of Kerr-like medium on the dynamics of two-level atom [47]. H. R. Baghshahi and fellows described the effect of Kerr-like medium and Stark on the dynamics of the Jaynes-Cummings model [48].

In this present work, our main focus is to investigate the QFI and QE of moving two-level atom which is interacting with coherent field and thermal field in presence of ID, Kerr medium and Stark shift. We have calculated VNE and QFI of the two-level atomic system in the presence of atomic motion and without atomic motion. Here, we use the QFI, based on symmetric logarithmic derivative (SLD) operator, to quantify entanglement of moving two-level atom interacting with a single-mode thermal field under the influence of ID. This study mainly investigates the time evolution of QFI and VNE under ID for moving and non moving two-level atomic system interacting with a thermal field. The time evolution of the wave function of the complete system is numerically evaluated. Then, the dynamics of the two-level atom by considering "QFI" and VNE are 
discussed, in detail. It is deduced from the numerical results that, both the ID and thermal environment play an important role during the time evolution of the quantum system. QFI and QE show an opposite response during its time evolution in the presence of the thermal environment. The time evolution of QFI is found to be highly sensitive to ID as compared to the QE. Furthermore, the degree of entanglement changes drastically when we increase the ID parameter in the absence of atomic motion. The increased damping behaviour of QE validates the basic fact that the system is more prone to intrinsic decoherence for larger time-scales. We study the dynamics of QE and QFI for the two-level atomic system under the influence of Stark shift and Kerr medium. The time evolution of QFI and QE for a two-level atomic system influenced by Stark effect and Kerr-like medium is investigated. It is seen that Stark and Stark and Kerr's medium plays a dominent during the time evolution of the quantum system. Dynamics of QFI are heavily influenced under the effect of linear Kerr medium as compared to the QE. Whereas the effect of non-linear Kerr medium is more prominent on the QE instead of QFI. Similarly, the Stark effect strongly influences the QE of the two-level atomic system. The QFI and QE evolve with time as we increase the Stark effect parameter. Finally, the quantum system is found highly sensitive to these environmental influences.

The organization of the paper is as follows: In Section 2, we describe the brief introduction of QFI and VNE and their formulas related to numerical calculations. The Hamiltonian of the system and dynamics are presented in Section 3. In Section 4, we present detailed results and numerical discussions. In Section 5, we present a brief conclusion.

\section{Quantum Fisher Information (QFI) of Bipartite System}

Von Neumann entropy (VNE) is the most important and basic QE measurement tool when the quantum system is in the pure state, therefore VNE is used to measure QE between the field and the two atoms. The VNE is presented in the form of eigenvalues of the atomic density matrix as [49] [50]

$$
S_{A B}=-\operatorname{Tr}\left(\rho_{A B} \ln \rho_{A B}\right)=-\sum_{i=1}^{2} r_{i} \ln r_{i},
$$

where $r_{i}$ are the eigenvalues of the atomic density matrix $\rho_{A B}$.

The QFI gives the maximum information about the estimated parameter and the QFI which is related to $\theta$ can be represented as [51] [52] [53]

$$
I_{\theta}=\sum_{k} \frac{\left(\partial_{\theta} \lambda_{k}\right)^{2}}{\lambda_{k}}+2 \sum_{k, k^{\prime}} \frac{\left(\lambda_{k}-\lambda_{k^{\prime}}\right)^{2}}{\lambda_{k}+\lambda_{k^{\prime}}}\left|\left\langle k \mid \partial_{\theta} k^{\prime}\right\rangle\right|^{2},
$$

where $\lambda_{k}>0$ and $\lambda_{k}+\lambda_{k^{\prime}}>0$. The first term in Equation (2) is classical Fisher information (CFI) and the second term represents its quantum counterpart. Therefore, we can define the QFI of a bipartite density operator $\rho_{A B}$ in terms of $\theta$ as [54]

$$
I_{Q F}(t)=I_{A B}(\theta, t)=\operatorname{Tr}\left[\rho_{A B}(\theta, t)\left\{L^{2}(\theta, t)\right\}\right],
$$


where $L(\theta, t)$ is the quantum score [55] or the symmetric logarithmic derivative (SLD) which can be defined as

$$
\frac{\partial \rho_{A B}(\theta, t)}{\partial \theta}=\frac{1}{2}\left[L(\theta, t) \rho_{A B}(\theta, t)+\rho_{A B}(\theta, t) L(\theta, t)\right] .
$$

\section{Intrinsic Decoherence Model}

In real practical situations, any quantum system which is said to be open can never be completely isolated from the environment. Milburn [56] gives a simple model of ID by making a modification in standard quantum mechanics. $\mathrm{He}$ supposed that on a sufficiently small time scale $\tau$, the system state takes the form

$$
\rho(t+\tau)=\exp \left[-\frac{i}{\hbar} \theta(\tau) H\right] \rho(t) \exp \left[\frac{i}{\hbar} \theta(\tau) H\right],
$$

with a probability of $\rho(t)$. The master equation presenting the ID under the Markovian approximations is given by

$$
\frac{\mathrm{d} \rho(t)}{\mathrm{d} t}=-i[H, \rho(t)]-\frac{\gamma}{2}[H,[H, \rho(t)]],
$$

where $\gamma$ is the ID parameter. The above master equation can be expressed by the solution as given below

$$
\rho(t)=\sum_{k=0}^{\infty} \frac{(\gamma t)^{k}}{k !} M^{k} \rho(0) M^{\dagger k},
$$

where $\rho(0)$ is the density operator of the initial state and $M^{k}$ is defined as

$$
M^{k}=H^{k} \mathrm{e}^{-i H t} \mathrm{e}^{-\frac{\gamma t}{2} H^{2}} .
$$

\section{The System Hamiltonian and Its Dynamics}

We have considered a system of moving two-level atom interacting with a coherent and thermal field as shown in Figure 1.

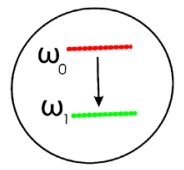

Two Level Cascade System

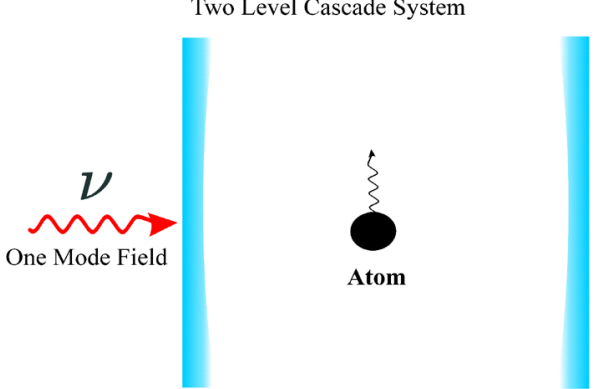

Figure 1. (Systematic diagram) Interaction of two-level atom with the single-mode coherent field. 
The total Hamiltonian of the system $\hat{H}_{T}$ under the RWA for a particular system can be described as [57]

$$
\hat{H}_{T}=\hat{H}_{\text {Atom-Field }}+\hat{H}_{I},
$$

where $\hat{H}_{\text {Atom-Field }}$ is representing the Hamiltonian for the non-coupling atom and field, and the interaction part is given by $\hat{H}_{I}$. We will write $\hat{H}_{\text {Atom-Field }}$ as

$$
\hat{H}_{\text {Atom-Field }}=\sum_{j} \omega_{j} \hat{\sigma}_{j, j}+v \hat{a}^{\dagger} \hat{a},
$$

where $\hat{\sigma}_{j, j}=|j\rangle\langle j|$ are describing as population operators for the jth level and $v$ is the frequency of oscillation.

The interaction Hamiltonian a of two-level atomic system for the case which is not resonant is written as [58]

$$
\hat{H}_{I}=\sum_{s=1}^{1} \Omega(t)\left[\hat{a} \mathrm{e}^{-i \Delta_{s} t}\left(\hat{\sigma}_{s, s+1}\right)+\left(\hat{a} \mathrm{e}^{-i \Delta_{s} t}\left(\hat{\sigma}_{s, s+1}\right)\right)^{\dagger}\right] .
$$

We can define the detuning parameter as

$$
\Delta_{s}=v-\left(\omega_{s}-\omega_{s+1}\right) .
$$

We have also considered the case in which the moving two-level atom interacting with a coherent field in the presence of non-linear Kerr-Like medium as shown in Figure 2.

$$
\hat{H}_{I}=\sum_{s=1}^{1} \Omega(t)\left[\hat{a} \mathrm{e}^{-i \Delta_{s} t}\left(\hat{\sigma}_{s, s+1}\right)+\left(\hat{a} \mathrm{e}^{-i \Delta_{s} t}\left(\hat{\sigma}_{s, s+1}\right)\right)^{\dagger}\right]+\chi\left(\hat{a}^{\dagger} \hat{a}\right)^{2},
$$

and when the Stark effect is included in the interaction Hamiltonian, it can be written as

$$
\hat{H}_{I}=\sum_{s=1}^{1} \Omega(t)\left[\hat{a} \mathrm{e}^{-i \Delta_{s} t}\left(\hat{\sigma}_{s, s+1}\right)+\left(\hat{a} \mathrm{e}^{-i \Delta_{s} t}\left(\hat{\sigma}_{s, s+1}\right)\right)^{\dagger}\right]+\beta \hat{a}^{\dagger} \hat{a}|g\rangle\langle g|,
$$

where $|g\rangle$ is representing the ground state of two-level atom and the coupling constant for atom and field is $G, \Omega(t)$ represents the shape function of the cavity-field mode [59] and the atomic motion is along $z$-axis. A realization of particular interest is

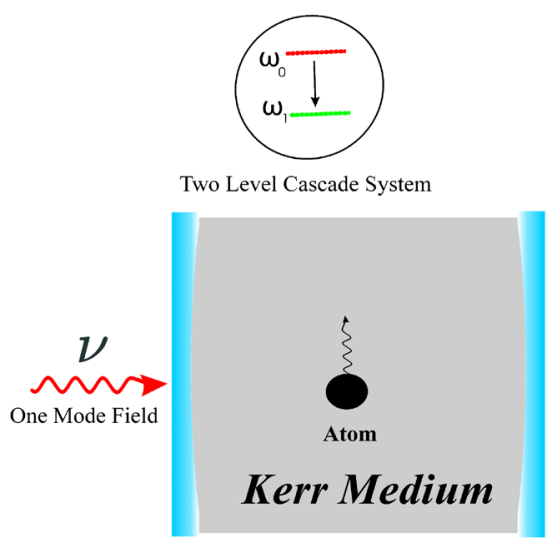

Figure 2. (Systematic diagram) Interaction of two-level atom with the single-mode coherent field in the presence of Kerr-like medium. 


$$
\begin{aligned}
& \Omega(t)=G \sin (\eta \pi v t / L) \text { in the presence of atomic motion, } \eta \neq 0, \\
& \Omega(t)=G \text { in the absence of atomic motion } \eta=0,
\end{aligned}
$$

where $v$ denotes the atomic motion velocity and $\eta$ is equal to the number of half wavelengths of the mode in the cavity and $L$ represents the cavity length in the $z$-direction. The atomic motion velocity is given as $v=G L / \pi$ which leads to

$$
\begin{aligned}
\Omega_{1}(t) & =\int_{0}^{t} \Omega(\tau) \mathrm{d} \tau=\frac{1}{\eta}(1-\cos (\eta \pi v t / L)) \text { for } \eta \neq 0, \\
& =G t \text { for } \eta=0 .
\end{aligned}
$$

The optimal input state after the phase gate operation for a two-level atomic system interacting with a single-mode cavity field can be written as

$$
|\Psi(0)\rangle=\frac{1}{\sqrt{2}}\left(|1\rangle+\mathrm{e}^{i \phi}|0\rangle\right) \otimes|\alpha\rangle,
$$

where $|1\rangle$ is the ground state and $|0\rangle$ is the excited state of an atom and $\alpha$ is the coherent state of the input field given as

$$
|\alpha\rangle=\sum_{n=0}^{\infty} \alpha^{n} \sqrt{\mathrm{e}^{-|\alpha|^{2}} / n !}|n\rangle .
$$

The optimal input state for a two-level atomic system interacting with a thermal field can be written as

$$
\rho=\rho(0) \otimes \rho_{f}(0),
$$

where $\rho(0)=|\Psi(0)\rangle\langle\Psi(0)|$ and $\rho_{f}(0)$ is the state of input thermal field. We have used thermal state of the field as input field state in the atom field interaction of the two-level atomic system which is given as

$$
\rho_{f}(0)=\sum_{n=0}^{\infty} P(n)|n\rangle\langle n| .
$$

where $|n\rangle$ is the fock state.

$$
P(n)=\frac{\bar{n}^{n}}{(\bar{n}+1)^{(n+1)}},
$$

where $\bar{n}$ is the mean photon number and is given as

$$
\bar{n}=\frac{1}{\mathrm{e}^{\hbar \omega_{f} / k_{B} T}-1},
$$

where $k_{B}$ is the Boltzmann constant, $\omega_{f}$ is the frequency of the cavity mode and $T$ is the temperature.

The wave function $|\Psi(t)\rangle$ in terms of unitary time evolution operator $\hat{U}(t)$ can be written as

$$
|\Psi(t)\rangle=\hat{U}(t)|\Psi(0)\rangle
$$

where $\hat{U}(t)$ is given by

$$
U(t)=\sum_{z=1}^{2} \exp \left(E_{z} t\right)\left|\varphi_{z}(t)\right\rangle\left\langle\varphi_{z}(t)\right|,
$$


where $\left|\varphi_{z}(t)\right\rangle$ and $E_{z}(t)$ are eigenvectors and eigenvalues of the Hamiltonian $H_{I}$, respectively and $\gamma$ is the ID parameter. The QFI is calculated numerically for the atom-field density matrix as given below

$$
\hat{\rho}(t)=\hat{U}(t) \rho(0) \hat{U}^{\dagger}(t) .
$$

One can write the explicit expression of the density matrix as

$$
\hat{\rho}(t)=\sum_{m, n}^{2}\left|\Psi_{n}(t)\right\rangle\left\langle\Psi_{n}(t)|\hat{\rho}(t)| \Psi_{m}(t)\right\rangle\left\langle\Psi_{m}(t)\right| .
$$

The density matrix can also be written as

$$
\hat{\rho}(t)=\sum_{m n} \exp \left(-\frac{\gamma t}{2}\left(E_{m}-E_{n}\right)^{2}-i\left(E_{m}-E_{n}\right) t\right) \times\left\langle\Psi_{m}|\hat{\rho}(0)| \Psi_{n}\right\rangle\left|\Psi_{m}\right\rangle\left\langle\Psi_{n}\right|,
$$

where $E_{m, n}$ and $\Psi_{m, n}$ are the eigenvalues and the corresponding eigenvectors of $H_{I}$ respectively. Now, the effect of different environmental parameters $\gamma, \theta$ and $\eta$ on the dynamics of QFI and VNE is discussed in detail in the next section.

\section{Numerical Results and Discussions}

In this section, we will present the results of the time evolution of QFI and VNE of a system of the two-level atom interacting with a coherent field and thermal field under the influence of Stark, Kerr (non-linear), and ID effects. We scaled out the time $t$, for example, one unit of time is described by the inverse of the coupling constant G. Initially, we investigate the time evolution of QFI and VNE for two-level atomic system interacting with coherent and thermal fields under the influence of ID, a (non-linear) Kerr medium, and a Stark effect with and without atomic motion.

In Figure 3 and Figure 4, we plot the QFI and VNE as a function of time for the two-level atomic system interacting with a thermal field under the influence of ID for $|\alpha|^{2}=6, \gamma=0,0.0001$ and 0.001 for phase shift $\phi=0, \pi / 4$, and atomic motion parameter $\eta=0,1$. The gradual decay of QFI is observed in the thermal environment in the presence of ID. Particularly at value $\gamma=0.001$, both QFI and VNE decrease rapidly as compared to the value at $\gamma=0.0001$. So it means that as ID parameter increased there is a prominent decay of QE as compared to QFI. In the case of the thermal field in the presence of ID, We find that the QFI and QE show an opposite response during the time evolution when the atomic motion parameter is $\eta=1$. Periodic and opposite behaviour of QFI and VNE is observed in the presence of atomic motion. It is shown that the atomic motion destroys the monotonic correlation between QFI and QE. It is found that the presence of a thermal environment leads to the suppression of QFI during its time evolution. Furthermore, the effect of the thermal environment dominates the ID effect. Paying attention to the effect of the atomic motion parameter, it is observed that an increase of atomic motion parameter and ID results in the loss of the purity of the state of the system. The damping behaviour of QE is seen under ID for larger time-scales in the absence of atomic 


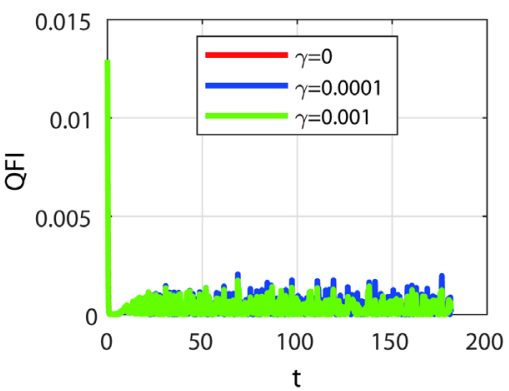

(a)

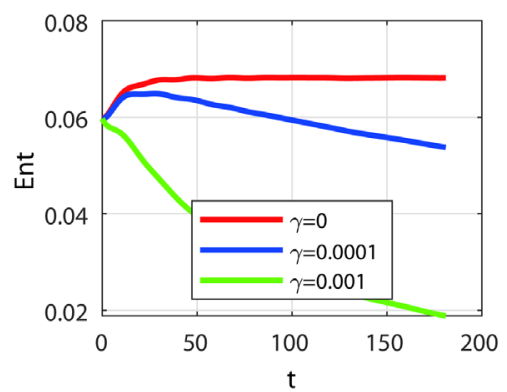

(c)

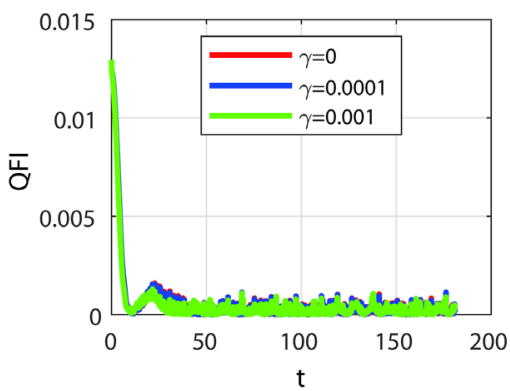

(b)

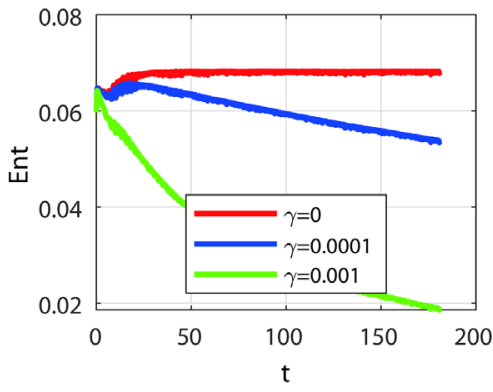

(d)

Figure 3. (Color online) The QFI (upper panel) and VNE (lower panel) as a function of time for two-level atom interacting with a thermal field for $\alpha=6$ in the presence ID parameter $\gamma=0,0.0001,0.001$ of and the phase shift estimator parameter $\varphi=0$ (left panel) and $\pi / 4$ (right panel). The atomic motion parameter $\eta$ is neglected. (a) $\phi=$ 0, 2-level; (b) $\phi=\pi / 4,2$-level; (c) $\phi=0,2$-level; (d) $\phi=\pi / 4,2$-level.

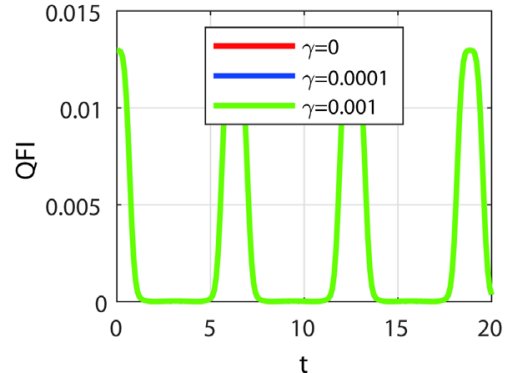

(a)

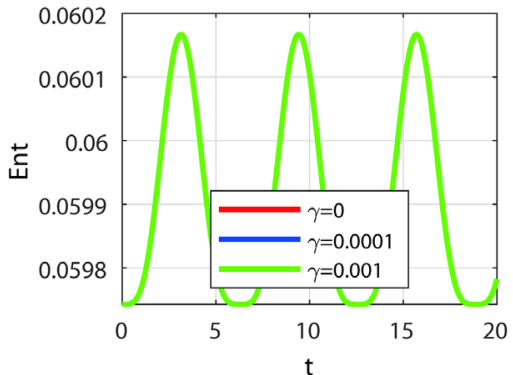

(c)

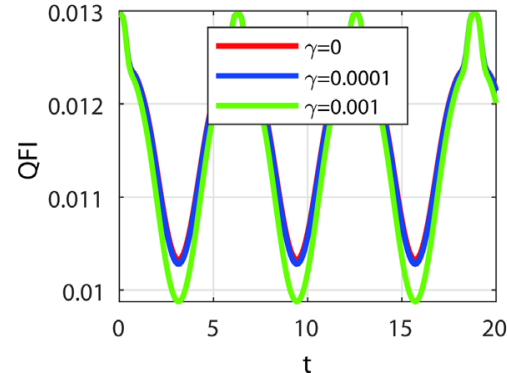

(b)

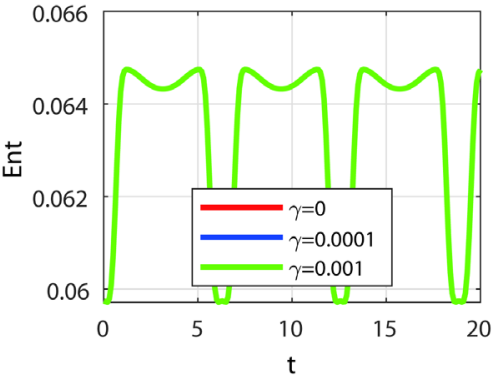

(d)

Figure 4. (Color online) The QFI (upper panel) and VNE (lower panel) as a function of time for two-level atom interacting with thermal for $\alpha=6$ in presence ID parameter $\gamma=0,0.0001,0.001$ and the phase shift estimator parameter $\varphi=0$ (left panel) and $\pi / 4$ (right panel), The atomic motion parameter is $\eta=1$. (a) $\phi=0$, 2-level; (b) $\phi=$ $\pi / 4$, 2-level; (c) $\phi=0,2$-level; (d) $\phi=\pi / 4,2$-level. 
motion. The periodic behaviour of QE in the presence of atomic motion becomes modest under environmental effects. The decay of the QE is suppressed in the presence of the thermal environment as compared to the ID. Therefore, the ID and thermal environment are found to suppress the nonclassical effects of the quantum system. However, QE and QFI saturate to a lower level for longer time-scales under the environmental influences. Finally, according to our numerical results, the decaying of the QE becomes faster in a thermal environment as we increased the value of ID parameter. Moreover, these results can be useful to perform long-distance quantum communication, especially when long-living $\mathrm{QE}$ is needed and the effect of environments cannot be neglected.

In Figure 5 and Figure 6, we plot the QFI and VNE as a function of time for two-level system interacting with coherent field for $|\alpha|^{2}=6$ and the phase shift parameter $\phi=0$ (left panel) and $\pi / 4$ (right panel) for different values of $\chi$ (Non-linear Kerr medium) with and without atomic motion, i.e. $\eta=0,1$. The effect of non-linear Kerr medium is found to be more prominent on the QE and QFI at $\chi=0.3$, QFI decreases as time evolves but VNE increases, so QFI and VNE are showing opposite behaviour. In the case of atomic motion, both QFI and VNE exhibit opposite and periodic behaviour in the presence of non-linear Kerr medium. At values $\chi=1,3$, the effect of non-linear Kerr is not very significant in the case of both QFI and VNE in the absence of atomic motion. The

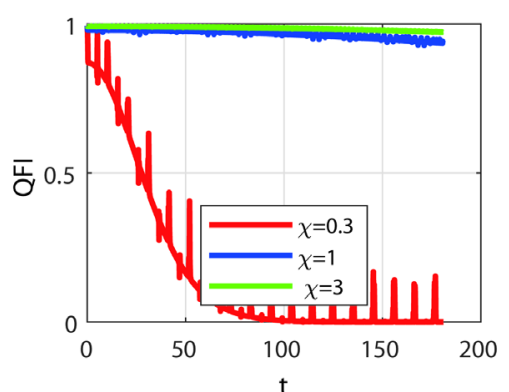

(a)

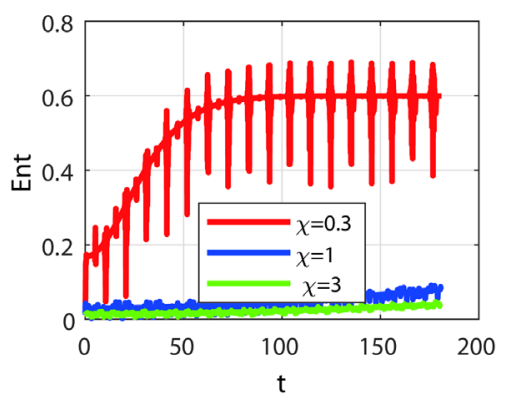

(c)

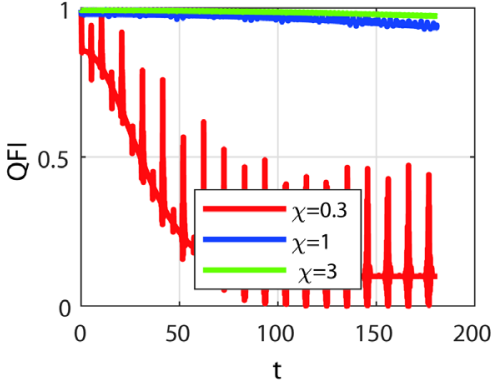

(b)

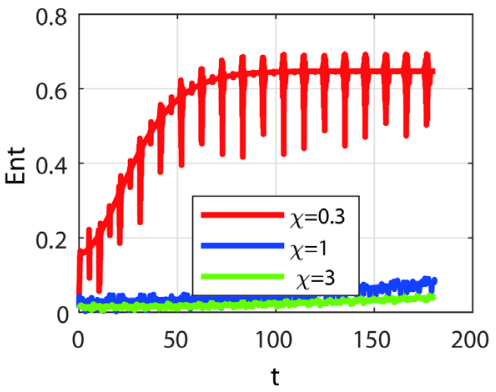

(d)

Figure 5. (Color online) The QFI (upper panel) and VNE (lower panel) as a function of time for a system of a two-level atom having interaction with a coherent field for $|\alpha|^{2}=6$ and the phase shift estimator parameter $\varphi=0$ (left panel) and $\pi / 4$ (right panel). The parameter $\eta$ of atomic motion is ignored and the value of $\chi=0.3,1,3$ (Non-linear Kerr). (a) $\phi=0,2$-level; (b) $\phi=\pi / 4,2$-level; (c) $\phi=0,2$-level; (d) $\phi=\pi / 4$, 2-level. 


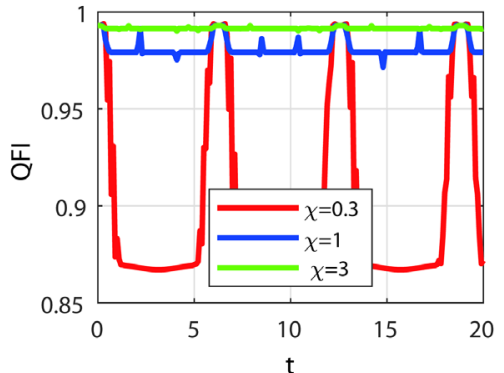

(a)

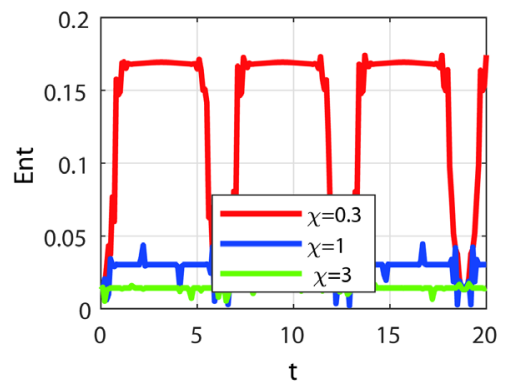

(c)

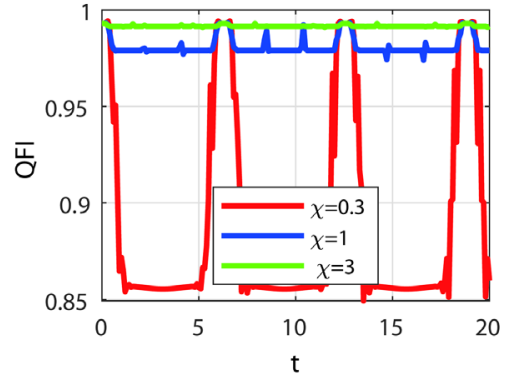

(b)

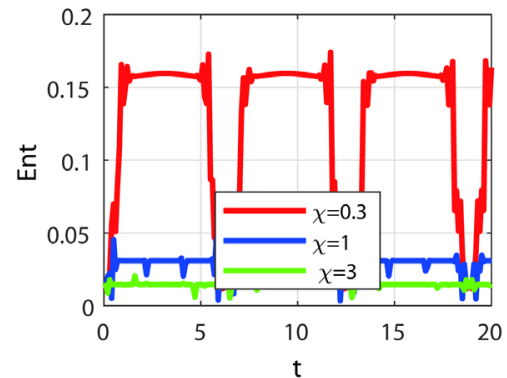

(d)

Figure 6. (Color online) The QFI (upper panel) and VNE (lower panel) as a function of time for a system of a two-level atom having interaction with a coherent field for $|\alpha|^{2}=6$ and the phase shift estimator parameter $\varphi=0$ (left panel) and $\pi / 4$ (right panel). The parameter $\eta$ of atomic motion is $\eta=1$ and the value of $\chi=0,1,3$ (Non-linear Kerr). (a) $\phi=0$, 2-level; (b) $\phi=\pi / 4,2$-level; (c) $\phi=0$, 2-level; (d) $\phi=\pi / 4$, 2-level.

effect of the non-linear Kerr medium is found to be more prominent on the QE as compared to the QFI in the absence of atomic motion. It is seen that the non-linear Kerr medium plays a dominant role during the time evolution of the quantum system. The periodic behavior of QFI and QE is further suppressed under the effect of the non-linear Kerr medium. These results show the strong dependence of QFI and QE on the non-linear Kerr medium. It is seen that at $\chi=0.3$ QFI decreases and VNE increases but this increasing and decreasing response becomes saturated as time evolves. At $\chi=1,3$, VNE decreases, but this decrease becomes saturated as time evolves. Hence, it is concluded that at higher values of the Kerr parameter, the QE decreases when compared with the smaller values. However, the non-linear Kerr medium has no prominent effect on QFI at higher and lower values of the Kerr parameter. In the presence of atomic motion, both QFI and VNE show periodic behavior.

In Figures 7-10 we plot QFI and VNE as a function of time for the two-level atomic system interacting with coherent field for $|\alpha|^{2}=6$ and the phase shift parameter $\phi=0$ (left panel) and $\pi / 4$ (right panel) for different values of $\beta=0.3,1,3$ (Stark shift) with and without atomic motion, i.e. $\eta=0,1$ respectively. The Stark effect is found to influence the QE of the two-level atomic system very strongly. Moreover, it has been seen that QFI and QE decay at the smaller values of the Stark parameter $(\beta=0.3)$ but as the value of the stark parameter is increased $(\beta=1,3)$, the QFI and QE show periodic behaviour even 


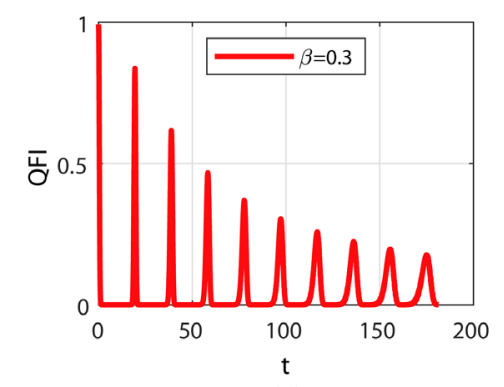

(a)

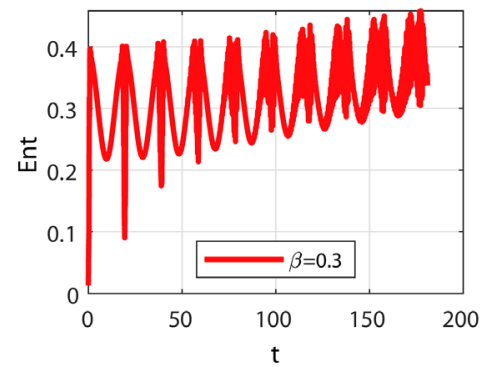

(c)

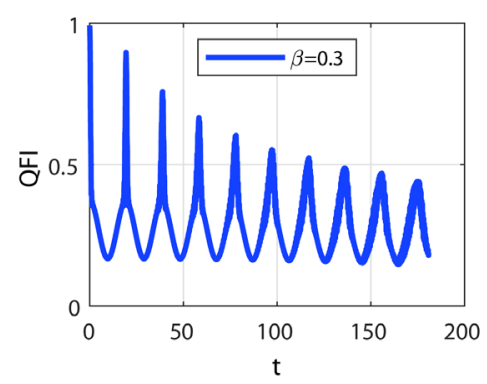

(b)

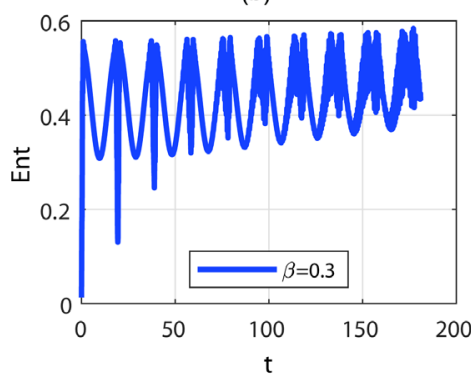

(d)

Figure 7. (Color online) The QFI (upper panel) and VNE (lower panel) as a function of time for a system of a two-level atom having interaction with a coherent field for $|\alpha|^{2}=6$ and the phase shift estimator parameter $\varphi=0 \quad$ (left panel) and $\pi / 4 \quad$ (right panel). The parameter $\eta$ of atomic motion is ignored and the value of $\beta=0.3$ (Stark effect). (a) $\phi=0$, 2-level; (b) $\phi=\pi / 4$, 2-level; (c) $\phi=0$, 2-level; (d) $\phi=\pi / 4$, 2-level.

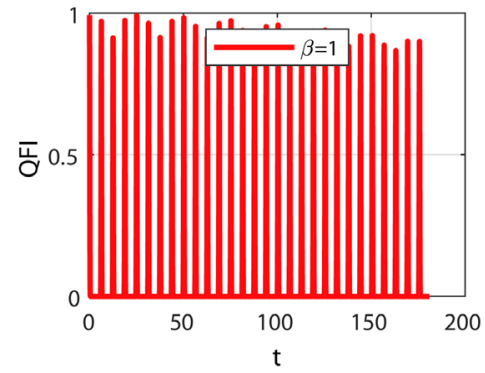

(a)

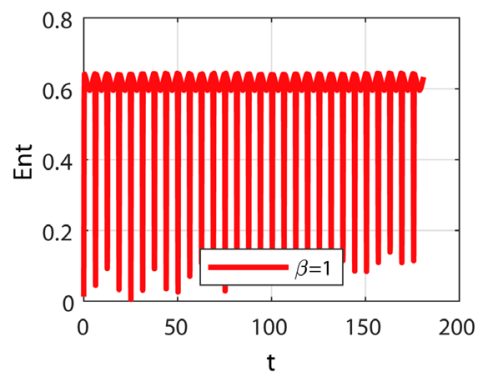

(c)

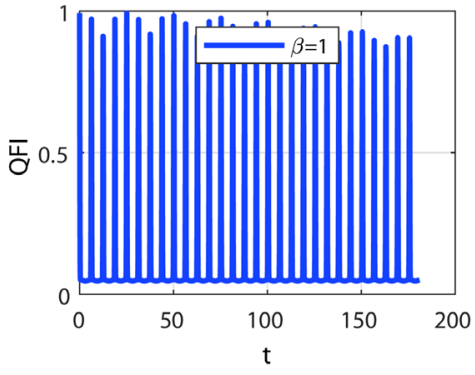

(b)

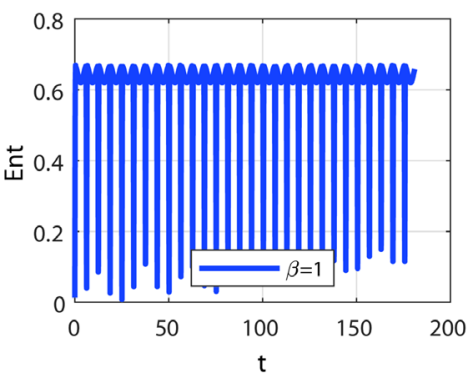

(d)

Figure 8. (Color online) The QFI (upper panel) and VNE (lower panel) as a function of time for a system of a two-level atom having interaction with a coherent field for $|\alpha|^{2}=6$ and the phase shift estimator parameter $\varphi=0$ (left panel) and $\pi / 4$ (right panel). The parameter $\eta$ of atomic motion is ignored and the value of $\beta=1$ (Stark effect). (a) $\phi$ =0, 2-level; (b) $\phi=\pi / 4,2$-level; (c) $\phi=0$, 2-level; (d) $\phi=\pi / 4,2$-level. 


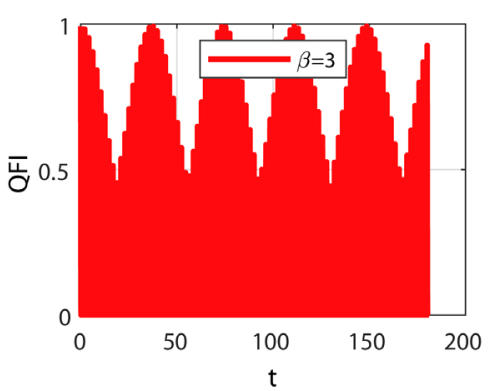

(a)

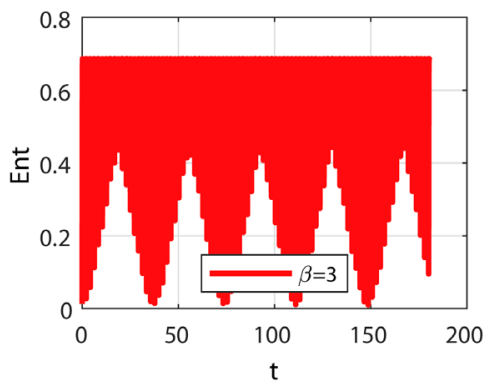

(c)

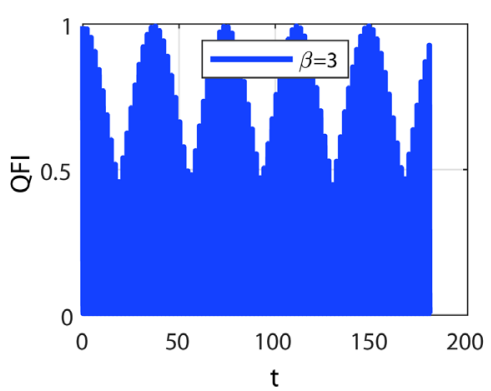

(b)

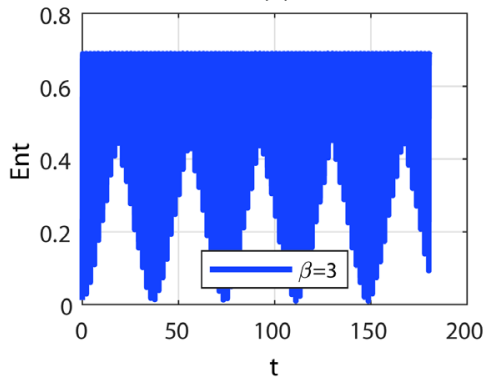

(d)

Figure 9. (Color online) The QFI (upper panel) and VNE (lower panel) as a function of time for a system of a two-level atom having interaction with a coherent field for $|\alpha|^{2}=6$ and the phase shift estimator parameter $\varphi=0 \quad$ (left panel) and $\pi / 4 \quad$ (right panel). The parameter $\eta$ of atomic motion is ignored and the value of $\beta=3$ (Stark effect). (a) $\phi$ =0,2-level; (b) $\phi=\pi / 4$, 2-level; (c) $\phi=0$, 2-level; (d) $\phi=\pi / 4$, 2-level.

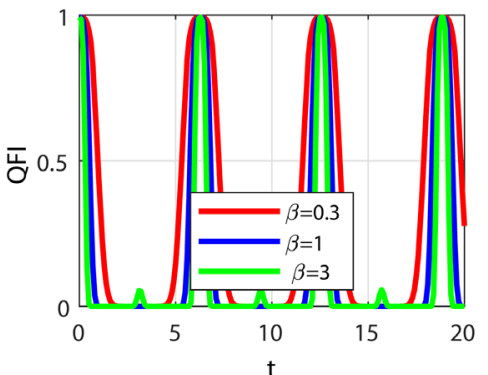

(a)

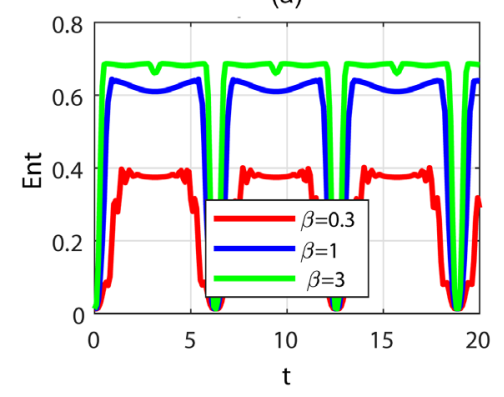

(c)

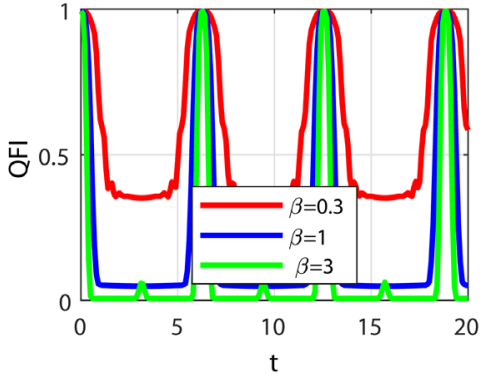

(b)

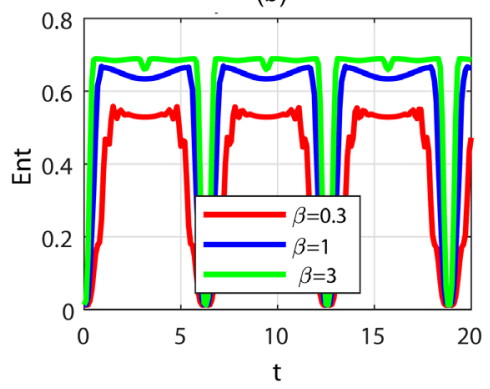

(d)

Figure 10. (Color online) The QFI (upper panel) and VNE (lower panel) as a function of time for a system of a two-level atom having interaction with a coherent field for $|\alpha|^{2}=6$ and the phase shift estimator parameter $\varphi=0 \quad$ (left panel) and $\pi / 4 \quad$ (right panel). The parameter $\eta$ of atomic motion is $\eta=1$ value of $\beta=0.3,1,3$ (Stark effect). (a) $\phi=$ 0, 2-level; (b) $\phi=\pi / 4,2$-level; (c) $\phi=0$, 2-level; (d) $\phi=\pi / 4$, 2-level. 
when the atomic motion is not present. Therefore, at higher values of $\beta$, the $\mathrm{QE}$ is sustained but at lower values of $\beta$, it decreases. In the presence of atomic motion, QFI and VNE show a periodic response at different $\beta$ values, so increasing the value of $\beta$ in the presence of atomic motion does not affect the QE. Finally, the quantum system is found highly sensitive to these environmental influences.

\section{Conclusions}

In summary, we have studied the dynamical evolution of QE and QFI for moving two-level atom interacting with the thermal field in the presence of ID. The time evolution of the entire system interacting with a thermal environment in the presence of ID is investigated numerically. It is seen that ID and thermal environment play an important and critical role in the time evolution of the quantum system. QFI and QE exhibit an opposite behaviour in a thermal environment in the presence of ID. It is observed that both QFI and QE are sensitive to ID in the presence of the thermal environment. It has been observed that QE drastically decreases when we increase the value of ID in the thermal field in the absence of atomic motion. The damping behaviour of QE is observed at larger time-scales at larger values of the ID parameter in the thermal field. The periodic behaviour of $\mathrm{QE}$ due to atomic motion becomes modest under the environmental effects. The ID and thermal environment are found to suppress the nonclassical effects of the quantum system. However, QE and QFI saturate to a lower level for longer time-scales under these environments. It ought to be mentioned that in the thermal environment decaying of the QE is faster at the larger values of ID. The results of our numerical calculations show that the two-level atomic system is more robust against the variations in the chosen parameters. Finally, it is revealed that the presented system can be useful for generating and maintaining $\mathrm{QE}$ in the presence of these environments in the presence of atomic motion. But in the case without atomic motion, the decay of $\mathrm{QE}$ is investigated under the influence of the thermal field and ID.

We have investigated the dynamics of QE and QFI for a two-level atomic system under the influence of the Stark shift and Kerr medium. The time evolution of QFI and QE for a two-level atomic system influenced by the Stark effect and Kerr-like medium is investigated. It is seen that Stark and Kerr's medium play a dominant role during the time evolution of the quantum system. The effect of non-linear Kerr medium is more prominent on the QFI and VNE at the smaller value of the Kerr parameter. As the value of the Kerr parameter is increased, the effect of non-linear Kerr does not remain significant on both QFI and QE. Similarly, the Stark effect strongly influences the QE of a two-level atomic system. Furthermore, it has been observed that QFI and QE decay soon at the smaller values of the Stark parameter but as the value of the Stark parameter is increased, the QFI and QE show periodic behaviour even when the atomic motion is not present. So it is concluded that at larger values of the Stark paprameter, the QE is maintained and sustained. QE and QFI exhibit opposite and periodic 
behaviour in the presence of atomic motion in case of linear, non-linear Kerr medium and stark effect. Finally, the quantum system is found highly sensitive to these environmental influences.

\section{Conflicts of Interest}

The authors declare no conflicts of interest regarding the publication of this paper.

\section{References}

[1] Helstrom, C.W. (1976) Quantum Detection and Estimation Theory. Academic, New York.

[2] Holevo, A.S. (1982) Probabilistic and Statistical Aspect of Quantum Theory. North-Holland, Amsterdam.

[3] Meyer, V., Rowe, M., Kielpinski, D., Sackett, C., Itano, W., Monroe, C. and Wineland, D.J. (2001) Experimental Demonstration of Entanglement-Enhanced Rotation Angle Estimation Using Trapped Ions. Physical Review Letters, 86, 5870-5873. https://doi.org/10.1103/PhysRevLett.86.5870

[4] Leibfried, D., Barrett, M., Schaetz, T., Britton, J., Chiaverini, J., Itano, W., Jost, J., Langer, C. and Wineland, D. (2004) Toward Heisenberg-Limited Spectroscopy with Multiparticle Entangled States. Science, 304, 1476-1478. https://doi.org/10.1126/science.1097576

[5] Afek, I., Ambar, O. and Silberberg, Y. (2010) High-NOON States by Mixing Quantum and Classical Light. Science, 328, 879-881. https://doi.org/10.1126/science.1188172

[6] Pezzé, L. and Smerzi, A. (2008) Mach-Zehnder Interferometry at the Heisenberg Limit with Coherent and Squeezed-Vacuum Light. Physical Review Letters, 100, Article ID: 073601. https://doi.org/10.1103/PhysRevLett.100.073601

[7] Giovannetti, V., Lloyd, S. and Maccone, L. (2011) Advances in Quantum Metrology. Nature Photonics, 5, 222-229. https://doi.org/10.1038/nphoton.2011.35

[8] Bollinger, J.J., Itano, W.M., Wineland, D.J. and Heinzen, D.J. (1996) Optimal Frequency Measurements with Maximally Correlated States. Physical Review A, 54, R4649. https://doi.org/10.1103/PhysRevA.54.R4649

[9] Hubner, M. (1992) Explicit Computation of the Bures Distance for Density Matrices. Physics Letters A, 163, 239-242. https://doi.org/10.1016/0375-9601(92)91004-B

[10] Braunstein, S.L. and Caves, C.M. (1994) Statistical Distance and the Geometry of Quantum States. Physical Review Letters, 72, 3439-3443. https://doi.org/10.1103/PhysRevLett.72.3439

[11] Petz, D. (1996) Monotone Metrics on Matrix Spaces. Linear Algebra and its Applications, 244, 81-96. https://doi.org/10.1016/0024-3795(94)00211-8

[12] Pezze, L. and Smerzi, A. (2009) Entanglement, Nonlinear Dynamics, and the Heisenberg Limit. Physical Review Letters, 102, Article ID: 100401. https://doi.org/10.1103/PhysRevLett.102.100401

[13] Ma, J. and Wang, X.G. (2009) Fisher Information and Spin Squeezing in the Lipkin-Meshkov-Glick Model. Physical Review A, 80, Article ID: 012318. https://doi.org/10.1103/PhysRevA.80.012318

[14] Rivas, A. and Luis, A. (2010) Precision Quantum Metrology and Nonclassicality in Linear and Nonlinear Detection Schemes. Physical Review Letters, 105, Article ID: 
010403. https://doi.org/10.1103/PhysRevLett.105.010403

[15] Giovannetti, V., Lloyd, S. and Maccone, L. (2006) Quantum Metrology. Physical Review Letters, 96, Article ID: 010401. https://doi.org/10.1103/PhysRevLett.96.010401

[16] Huegla, S.F., Macchiavello, C., Pellizzari, T., Ekert, A.K., Plenio, M.B. and Cirac, J.I. (1997) Improvement of Frequency Standards with Quantum Entanglement. Physical Review Letters, 79, 3865. https://doi.org/10.1103/PhysRevLett.79.3865

[17] Escher, B.M., de Matos Fillo, R.L. and Davidovich, L. (2011) General Framework for Estimating the Ultimate Precision Limit in Noisy Quantum-Enhanced Metrology. Nature Physics, 7, 406-411. https://doi.org/10.1038/nphys1958

[18] Uys, H. and Meystre, P. (2007) Quantum States for Heisenberg-Limited Interferometry. Physical Review A, 76, Article ID: 013804. https://doi.org/10.1103/PhysRevA.76.013804

[19] Helstrom, C.W. (1976) Quantum Detection and Estimation Theory. Academic, New York.

[20] Holevo, A.S. (1982) Probabilistic and Statistical Aspects of Quantum Theory. North-Holland, Amsterdam.

[21] Wootters, W.K. (1981) Statistical Distance and Hilbert Space. Physical Review D, 23, 357-362. https://doi.org/10.1103/PhysRevD.23.357

[22] Braunstein, S.L. and Caves, C.M. (1994) Statistical Distance and the Geometry of Quantum States. Physical Review Letters, 72, 3439-3443. https://doi.org/10.1103/PhysRevLett.72.3439

[23] Lu, X.M., Wang, X.G. and Sun, C.P. (2010) Quantum Fisher Information Flow and Non-Markovian Processes of Open Systems. Physical Review A, 82, Article ID: 042103. https://doi.org/10.1103/PhysRevA.82.042103

[24] von Neumann, J. (1955) Mathematical Foundations of Quantum Mechanics. Princeton University, Princeton.

[25] Phoenix, S.J. and Knight, P.L. (1988) Fluctuations and Entropy in Models of Quantum Optical Resonance. Annals of Physics, 186, 381. https://doi.org/10.1016/0003-4916(88)90006-1

[26] Rungta, P., Buzek, V., Caves, C.M., Hillery, M. and Milburn, G.J. (2001) Universal State Inversion and Concurrence in Arbitrary Dimensions. Physical Review A, 64, Article ID: 042315. https://doi.org/10.1103/PhysRevA.64.042315

[27] Uhlmann, A. (2000) Fidelity and Concurrence of Conjugated States. Physical Review A, 62, Article ID: 032307. https://doi.org/10.1103/PhysRevA.62.032307

[28] Berrada, K., El Baz, M., Saif, F., Hassouni, Y. and Mnia, S. (2009) Entanglement Generation from Deformed Spin Coherent States Using a Beam Splitter. Journal of Physics A: Mathematical and Theoretical, 42, Article ID: 285306. https://doi.org/10.1088/1751-8113/42/28/285306

[29] Obada, A.-S.F. and Abdel-Khalek, S. (2010) Entanglement Evaluation with Atomic Fisher Information. Physica A, 389, 891-898. https://doi.org/10.1016/j.physa.2009.09.015

[30] Abdel-Khalek, S. (2009) Dynamics of Fisher Information in Kerr Medium. International Journal of Quantum Information, 7, 1541. https://doi.org/10.1142/S0219749909005870

[31] Sergent, M., Scully, M.O. and Lamb, W.E. (1974) Laser Physics. Adison-Wesely, Reading.

[32] Allen, L. and Eberly, J.H. (1975) Optical Resonance and Two-Level Atoms. Wiley, 
New York.

[33] Jaynes, E.T. and Cummings, F.W. (1963) Comparison of Quantum and Semiclassical Radiation Theories with Application to the Beam Maser. Proceedings of the IEEE, 51, 89. https://doi.org/10.1109/PROC.1963.1664

[34] Narozhny, N.B., Sanchez, J.J. and Eberly, J.H. (1981) Coherence versus Incoherence: Collapse and Revival in a Simple Quantum Model. Physical Review A, 23, 236. https://doi.org/10.1103/PhysRevA.23.236

[35] Buck, B. and Sukumar, C.V. (1981) Exactly Soluble Model of Atom-Phonon Coupling Showing Periodic Decay and Revival. Physics Letters A, 81, 132-135. https://doi.org/10.1016/0375-9601(81)90042-6

[36] Abdel-Hafez, A.M., Obada, A.S.F. and Ahmed, M.M.A. (1987) N-Level Atom and (N-1) Modes: An Exactly Solvable Model with Detuning and Multiphotons. Journal of Physics A, 20, L359. https://doi.org/10.1088/0305-4470/20/6/004

[37] Law, C.W. and Eberly, J.H. (1991) Response of a Two-Level Atom to a Classical Field and a Quantized Cavity Field of Different Frequencies. Physical Review, 43, 6337-6344. https://doi.org/10.1103/PhysRevA.43.6337

[38] Abdel-Aty, M., Abdel-Khalek, S. and Obada, A.S.F. (2000) Pancharatnam Phase of Two-Mode Optical Fields with Kerr Nonlinearity. Optical Review, 7, 499-504. https://doi.org/10.1007/s10043-000-0499-6

[39] Abdel-Aty, M., Abdel-Khalek, S. and Obada, A.-S.F. (2001) Entropy Evolution of the Bimodal Field Interacting with an Effective Two-Level Atom via the Raman Transition in Kerr Medium. Chaos, Solitons \& Fractals, 12, 2015-2022. https://doi.org/10.1016/S0960-0779(00)00116-8

[40] Faghihi, M.J. and Tavassoly, M.K. (2012) Dynamics of Entropy and Nonclassical Properties of the State of a $\Lambda$-Type Three-Level Atom Interacting with a Single-Mode Cavity Field with Intensity-Dependent Coupling in a Kerr Medium. Journal of Physics B: Atomic, Molecular and Optical Physics, 45, Article ID: 035502. https://doi.org/10.1088/0953-4075/45/3/035502

[41] Abdalla, M.S., Obada, A.-S.F. and Abdel-khalek, S. (2008) Entropy Squeezing of Time Dependent Single-Mode Jaynes-Cummings Model in Presence of Non-Linear Effect. Chaos, Solitons and Fractals, 36, 405-417.

https://doi.org/10.1016/j.chaos.2006.06.067

[42] Ateto, M.S. (2010) Quantum Entropy of a Nonlinear Two-Level Atom with Atomic Motion. International Journal of Theoretical Physics, 49, 276-292. https://doi.org/10.1007/s10773-009-0201-0

[43] He, Z., Xiong, Z. and Zhang, Y. (2006) Influence of Intrinsic Decoherence on Quantum Teleportation via Two-Qubit Heisenberg XYZ Chain. Physics Letters A, 354, 79-83. https://doi.org/10.1016/j.physleta.2006.01.038

[44] Abdel-Aty, Y. (2008) New Features of Total Correlations in Coupled Josephson Charge Qubits with Intrinsic Decoherence. Physics Letters A, 372, 3719-3724. https://doi.org/10.1016/j.physleta.2008.02.017

[45] Abdel-Khalek, S., Berrada, K. and Obada, A.S.F. (2012) Quantum Fisher Information for a Single Qubit System. The European Physical Journal D, 66, Article No. 69. https://doi.org/10.1140/epjd/e2012-20576-8

[46] Berrada, K., Abdel-Khalek, S. and Obadad, A.-S.F. (2012) Quantum Fisher Information for a Qubit System Placed inside a Dissipative Cavity. Physics Letters A, 376, 1412-1416. https://doi.org/10.1016/j.physleta.2012.03.023

[47] Buzek, V. and Jex, I. (1990) Dynamics of a Two-Level Atom in a Kerr-Like Medium. 
Optics Communications, 78, 425-435. https://doi.org/10.1016/0030-4018(90)90340-Y

[48] Baghshahi, H.R., Tavassoly, M.K. and Behjat, A. (2014) Entropy Squeezing and Atomic Inversion in the k-Photon Jaynes-Cummings Model in the Presence of the Stark Shift and a Kerr Medium: A Full Nonlinear Approach. Chinese Physics B, 23, Article ID: 074203. https://doi.org/10.1088/1674-1056/23/7/074203

[49] Abdel-Khalek, S. and Halawani, S.H.A. (2016) New Features of the Stationary and Moving Atom-Atom Entanglement. Optik-International Journal for Light and Electron Optics, 127, 9020-9025. https://doi.org/10.1016/j.ijleo.2016.05.102

[50] von Neumann, J. (1955) Mathematical Foundations of Quantum Mechanics. Princeton University Press, Princeton.

[51] Zhong, W., Sun, Z., Ma, J., Wang, X.G. and Nori, F. (2013) Fisher Information under Decoherence in Bloch Representation. Physical Review A, 87, Article ID: 022337. https://doi.org/10.1103/PhysRevA.87.022337

[52] Berrada, K., Khalek, S.A. and Ooi, C.H.R. (2012) Quantum Metrology with Entangled Spin-Coherent States of Two Modes. Physical Review A, 86, Article ID: 033823. https://doi.org/10.1103/PhysRevA.86.033823

[53] Berrada, K. (2013) Quantum Metrology with SU $(1,1)$ Coherent States in the Presence of Nonlinear Phase Shifts. Physical Review A, 88, Article ID: 013817. https://doi.org/10.1103/PhysRevA.88.013817

[54] Lu, X., Wang, X. and Sun, C.P. (2010) Quantum Fisher Information Flow and Non-Markovian Processes of Open Systems. Physical Review A, 82, Article ID: 042103. https://doi.org/10.1103/PhysRevA.82.042103

[55] Barndorff-Nielsen, O.E., Gill, R.D. and Jupp, P.E. (2003) On Quantum Statistical Inference. Journal of the Royal Statistical Society: Series B, 65, 775-816. https://doi.org/10.1111/1467-9868.00415

[56] Milburn, G.J. (1991) Intrinsic Decoherence in Quantum Mechanics. Physical Review A, 44, 5401-5406. https://doi.org/10.1103/PhysRevA.44.5401

[57] Plenio, M.B. and Knight, P.L. (1997) Decoherence Limits to Quantum Computation Using Trapped Ions. Proceedings of the Royal Society of London. Series A, 453, 2017-2041. https://doi.org/10.1098/rspa.1997.0109

[58] Kuang, L.M., Chen, X. and Ge, M.L. (1995) Influence of Intrinsic Decoherence on Nonclassical Effects in the Multiphoton Jaynes-Cummings Model. Physical Review A, 52, 1857-1869. https://doi.org/10.1103/PhysRevA.52.1857

[59] Enaki, N.A. and Ciobanu, N.J. (2008) Horopter Measured as a Function of Wavelength. Journal of Modern Optics, 55, 589-598.

https://doi.org/10.1080/09500340701470029 